Sādhanā Vol. 36, Part 4, August 2011, pp. 463-488. (C) Indian Academy of Sciences

\title{
Efficient explicit formulation for practical fuzzy structural analysis
}

\author{
A S BALU and B N RAO* \\ Structural Engineering Division, Department of Civil Engineering, \\ Indian Institute of Technology Madras, Chennai 600 036, India \\ e-mail: bnrao@iitm.ac.in
}

MS received 8 August 2010; revised 4 February 2011; accepted 7 June 2011

\begin{abstract}
This paper presents a practical approach based on High Dimensional Model Representation (HDMR) for analysing the response of structures with fuzzy parameters. The proposed methodology involves integrated finite element modelling, HDMR based response surface generation, and explicit fuzzy analysis procedures. The uncertainties in the material, geometric, loading and structural parameters are represented using fuzzy sets. To facilitate efficient computation, a HDMR based response surface generation is employed for the approximation of the fuzzy finite element response quantity.
\end{abstract}

Keywords. Fuzzy analysis; finite element; high dimensional model representation; response surface; explicit formulation.

\section{Introduction}

The exponential growth of computational power during the last few decades has enabled the finite element (FE) analysis of many real-life engineering systems which are too complex to be analytically solved in a closed form. In the traditional deterministic finite element analysis, system parameters such as mass, geometry and material properties are assumed to be known precisely and defined exactly. However, in practice most of the data used in the solution process of many practical engineering systems are either collected from experiments or acquired as empirical data from the past, which are usually ill defined, imprecise and uncertain in nature. The uncertainty present in the problem can be categorized into randomness and fuzziness.

Probabilistic methods are especially suitable in case of uncertainty due to randomness for which the information on both the range and the probability density function are available. The probabilistic concept is already well-established for the extension of the deterministic finite element method towards uncertainty assessment. This has led to a number of probabilistic finite element procedures (Haldar \& Mahadevan 2000; Lernout et al 2006; Pellissetti \& Schueller 2007; Schueller et al 2004; Soize 2009) that are often used in conjunction with Monte Carlo

*For correspondence 
simulations. However, the use of a probabilistic approach can lead to subjective results if the amount of statistical data on the uncertainties is limited, and fuzzy information that is vague, imprecise, qualitative, linguistic or incomplete need to be included (Elishakoff 1995). The need to incorporate subjective information into the design and analysis of engineering systems has led to the development of fuzzy finite element methodology that began in the early 1990s.

Fuzzy set theory initiated by Zadeh (1965) provides a concept for the description of linguistic or subjective knowledge and incomplete data in a non-probabilistic manner. The use of Zadeh's concept in the finite element context has led to the development of the fuzzy finite element method for the solution of engineering problems (Dhingra et al 1992; Valliappan \& Pham 1995; Chen \& Rao 1997; Akpan et al 2001a, b). Its aim is to obtain the membership function of an output quantity, based on the fuzzy description of the uncertain input parameters. By using the $\alpha$-level technique, the interval finite element method forms the core of the fuzzy procedure (Gersem et al 2007). Over the last decade, several interval FE procedures have been reported, such as the vertex method (Dong \& Shah 1987; Hanss 2002; Donders et al 2005), the global optimization approaches (Valliappan \& Pham 1995; Möller et al 2000; Farkas et al 2008) and the interval arithmetic approaches (Rao \& Berke 1997; Qiu \& Elishakoff 1998; Dessombz et al 2001; Muhanna \& Mullen 2001). However, each of these techniques has its specific limitations when applied to realistic engineering problems, and none of them has been established as standard interval procedure, and the choice of technique depends on the type of the conducted FE analysis.

This paper presents a practical approach based on High Dimensional Model Representation (HDMR) (Li et al 2001a, b; Sobol 2003; Chowdhury et al 2008; Rao \& Chowdhury 2008) for analysing the response of structures with fuzzy parameters. The proposed methodology involves integrated finite element modelling, HDMR based response surface generation, and explicit fuzzy analysis procedure. The uncertainties in the material, geometric, loading and structural parameters are represented using fuzzy sets. To facilitate efficient computation, a HDMR based response surface generation is employed for the approximation of the fuzzy finite element response quantity, and the response is expressed in a separable closed-form expression as a linear combination of the fuzzy variables through the definition of intervening variables, then using the transformation technique the bounds on the response at each $\alpha$-level are obtained.

\section{Fuzzy finite element approach}

\subsection{Fuzzy sets}

The concept of fuzzy sets, introduced by Zadeh (1965), has gained an increasing popularity during the last two decades. Its most important property is that it is capable of describing linguistic and therefore incomplete information in a non-probabilistic manner. While a classical set clearly distinguishes between members and non-members, the fuzzy set introduces a degree of membership, represented by the membership function. For a fuzzy set $\tilde{x}$, the membership function $\mu_{\tilde{x}}(x)$ describes the grade of membership to the fuzzy set for each element $x$ in the domain $X$ :

$$
\tilde{x}=\left\{\left(x, \mu_{\tilde{x}}(x)\right) \mid(x \in X),\left(\mu_{\tilde{x}}(x) \in[0,1]\right)\right\} .
$$

If $\mu_{\tilde{x}}(x)=1, x$ is definitely a member of the set $\tilde{x}$, whereas if $\mu_{\tilde{x}}(x)=0, x$ is definitely not a member of the set $\tilde{x}$. For all $x$ with $0<\mu_{\tilde{x}}(x)<1$, the membership is not certain. The most frequently applied membership function shapes are the triangular and Gaussian (Ross 2004; Gersem et al 2007). 


\subsection{Fuzzy numerical analysis}

The fuzzy finite element method (FFEM) aims to obtain a fuzzy description of an FE analysis result, starting from the fuzzy descriptions of all non-deterministic FE model parameters. In practice, the description of uncertain parameters and quantities using fuzzy sets can be implemented using the $\alpha$-level strategy. This approach subdivides the membership function range into a number of $\alpha$-levels. The intersection with the membership function of the input uncertainties at each $\alpha$-level results in an interval $x_{i, \alpha}^{I}=\left[\underline{x}_{i}, \bar{x}_{i}\right]_{\alpha}$. With these input intervals of the $\alpha$-sublevel, an interval finite element (IFE) analysis is performed, resulting in an interval for the analysis result at the considered $\alpha$-level. Finally, the fuzzy solution is assembled from the resulting intervals at each sublevel.

The fuzzy finite element technique consists of the application of the $\alpha$-level strategy on the numerical procedure of the deterministic FE analysis. Through this procedure, the fuzzy FE analysis can be interpreted as a large-scale sensitivity analysis, which can be used to study the combined effect of the interval bounds of design variables on critical design properties. More comprehensive overview of fuzzy analysis for non-deterministic analysis in engineering design is available in reference (Moens \& Vandepitte 2004).

Through the $\alpha$-level strategy, the interval problem corresponding to the considered FE analysis forms the core of the fuzzy procedure. A number of general interval solution strategies have been proposed as the basis for fuzzy FE implementations. The most popular one is the interval arithmetic approach, in which all basic deterministic algebraic operations are replaced by their interval arithmetic counterparts. Although this approach is computationally very inexpensive, it is of little practical use as it tends to largely overestimate the interval outcome of each operation, accumulating in a huge amount of conservatism in the interval analysis result of realistic problems. Therefore, other approaches such as the vertex method (Dong \& Shah 1987; Dong \& Wong 1987; Hanss 2002; Donders et al 2005), the global optimization method (Valliappan \& Pham 1995; Möller et al 2000; Farkas et al 2008), and methods based on interval arithmetic (Rao \& Berke 1997; Qiu \& Elishakoff 1998; Dessombz et al 2001; Muhanna \& Mullen 2001) have been proposed for the solution of the underlying interval FE problem. Each of these techniques has its specific disadvantages when applied to realistic engineering problems, such that none of them has been established as standard interval procedure, and the choice of technique depends on the type of the conducted FE analysis.

The vertex method evaluates the function value at each of the vertices of the design space, represented by the bounds on the variables, to obtain the minimum and maximum values of the response. This approach requires $2^{N}$ function evaluations, where $N$ is the number of uncertain parameters. The easy implementation and the limited computational cost if the number of uncertainties is not too high form the major advantages of this method. The important limitation of the vertex method is that the exact interval results can be obtained only if the variation of the outputs is monotonic with respect to a variation of the uncertain parameters. This is difficult to predict in case of a general structural/mechanical problem. In case of nonlinear non-monotonic responses, the vertex method does not produce conservative results. This means that the real interval results are underestimated. Especially in design validation applications, this forms a severe drawback.

The global optimization techniques require the function value and/or gradient information at several points in the iterative process at each $\alpha$-cut, to obtain the minimum and maximum values of a nonlinear response. Taking into account the number of optimizations, the number of function evaluations required for one optimization and the computational cost of one function evaluation, this procedure is computationally expensive for most practical applications with implicit 
response functions. However, the computational efficiency of this approach can be improved by adopting the reduced optimization (Farkas et al 2008).

As an alternative to the optimization procedures, response surface based optimization methods (Akpan et al 2001a; Munck et al 2008) use a different approach: instead of evaluating the objective function every time during the optimization procedure, they first create an approximation of the objective function based on objective function evaluations in a number of so-called response points. This approximation, whose evaluation should be computationally less expensive than the evaluation of the objective function itself, is then used to find the optimum. Although more advanced approximation techniques such as Kriging methods (Jones et al 1998), neural networks (Chen 1996; Harvey 1994) and support vector regression (Drucker et al 1997) can be used for this application, response surfaces are chosen because they adequately describe the objective function behaviour. Response surface behaviour is easy to understand and to visualize, making it much easier to take corrective actions if the accuracy of the approximation is insufficient.

Response surface based optimization techniques prove to be extremely useful in the context of fuzzy analysis. A fuzzy analysis requires the same objective functions to be minimised and maximised on different $\alpha$-levels or, in optimization terms, with different bound constraints. Since the uncertain parameter intervals at higher $\alpha$-levels are subsets of the uncertain parameter interval at the lowest $\alpha$-level, a response surface suitable for the lowest $\alpha$-level can be used at all $\alpha$ levels. Since the construction of the response surfaces is the computationally most expensive part of these techniques, the computational cost of a fuzzy analysis is only slightly higher than the computational cost of an interval analysis based on fuzzy arithmetic operations. However, response surface based optimization techniques avoid other practical problems associated with interval analysis, which include the possibility of obtaining unbounded, unrealistic and nonunique solutions if the dependent relations between the fuzzy variables and the constrained fuzzy operation procedures are not considered in the formulation.

\section{Concept of HDMR and its importance to fuzzy structural analysis}

In recent years there have been efforts to develop efficient methods to approximate multivariate functions in such a way that the component functions of the approximation are ordered starting from a constant and gradually approaching to multivariance as we proceed along the terms like first-order, second-order and so on. One such method is High Dimensional Model Representation (HDMR) (Li et al 2001a, b; Sobol 2003). HDMR is a general set of quantitative model assessment and analysis tools for capturing the high-dimensional relationships between sets of input and output model variables. It is a very efficient formulation of the system response, if higher-order variable correlations are weak, allowing the physical model to be captured by the first few lower-order terms. Practically for most well-defined physical systems, only relatively low order correlations of the input variables are expected to have a significant effect on the overall response. HDMR expansion utilizes this property to present an accurate hierarchical representation of the physical system. The notion of 'high' dimensionality is system-dependent, with some situations being considered high for practical reasons at $N \simeq 3-5$, while others will only reach that level of complexity for $N>10$ or more. Recently potential of HDMR concepts is explored to find an equivalent continuous function to replace a univariate or multivariate piece-wise continuous function, rather than seeking an exact continuous function (Chowdhury et al 2008), and the HDMR concepts are extended to predict the failure probability of structural or mechanical systems subjected to random loads and material properties (Rao \& Chowdhury 2008). 
Let the $N$-dimensional vector $\boldsymbol{x}=\left\{x_{1}, x_{2}, \cdots, x_{N}\right\}$, represent the input variables of the model under consideration, and the response function as $g(\boldsymbol{x})$. Since the influence of the input variables on the response function can be independent and/or cooperative, HDMR expresses the response $g(\boldsymbol{x})$ as a hierarchical correlated function expansion in terms of the input variables as

$$
\begin{aligned}
g(\boldsymbol{x})= & g_{0}+\sum_{i=1}^{N} g_{i}\left(x_{i}\right)+\sum_{1 \leq i_{1}<i_{2} \leq N} g_{i_{1} i_{2}}\left(x_{i_{1}}, x_{i_{2}}\right)+\ldots \\
& +\sum_{1 \leq i_{1}<\ldots<i_{l} \leq N} g_{i_{1} i_{2} \ldots i_{l}}\left(x_{i_{1}}, x_{i_{2}}, \ldots, x_{i_{l}}\right)+\ldots+g_{12 \ldots N}\left(x_{1}, x_{2}, \ldots, x_{N}\right),
\end{aligned}
$$

where $g_{0}$ is a constant term representing the zeroth-order component function or the mean response of $g(\boldsymbol{x})$. The function $g_{i}\left(x_{i}\right)$ is a first-order term expressing the effect of variable $x_{i}$ acting alone, although generally nonlinearly, upon the output $g(\boldsymbol{x})$. The function $g_{i_{1} i_{2}}\left(x_{i_{1}}, x_{i_{2}}\right)$ is a second-order term which describes the cooperative effects of the variables $x_{i_{1}}$ and $x_{i_{2}}$ upon the output $g(\boldsymbol{x})$. The higher order terms give the cooperative effects of increasing numbers of input variables acting together to influence the output $g(\boldsymbol{x})$. The last term $g_{12, \cdots, N}\left(x_{1}, x_{2}, \cdots, x_{N}\right)$ contains any residual dependence of all the input variables locked together in a cooperative way to influence the output $g(\boldsymbol{x})$. Once all the relevant component functions in Equation 2 are determined and suitably represented, then the component functions constitute HDMR, thereby replacing the original computationally expensive method of calculating $g(\boldsymbol{x})$ by the computationally efficient model. Usually the higher order terms in Equation 2 are negligible such that HDMR with only few low order correlations, amongst the input variables are typically adequate in describing the output behaviour resulting in rapid convergence of HDMR expansion (Li et al 2001a). However, importance of higher order terms in HDMR is problem dependent and an independent analysis such as for example, the Sobol' sensitivity indices method (Sobol 1993) should be made beforehand to establish which order terms in the HDMR expansion are not important.

Depending on the method adopted to determine the component functions in Equation 2 there are two particular HDMR expansions: ANOVA-HDMR and cut-HDMR. ANOVA-HDMR is useful for measuring the contributions of the variance of individual component functions to the overall variance of the output. On the other hand, cut-HDMR expansion is an exact representation of the output $g(\boldsymbol{x})$ in the hyperplane passing through a reference point in the variable space.

In this work, cut-HDMR procedure is used to approximate the original response function with an explicit performance function to estimate the response of structural/mechanical systems subjected to fuzzy variables. With cut-HDMR method, first a reference point $\boldsymbol{c}=\left\{c_{1}, c_{2}, \cdots, c_{N}\right\}$ is defined in the variable space. In the convergence limit that all correlated functions in Equation 2 are considered, cut-HDMR is invariant to the choice of reference point $\boldsymbol{c}$. However, in practice the choice of reference point $\boldsymbol{c}$ is very important for cut-HDMR especially if only terms up to first- and second-order in Equation 2 are considered, and it is shown that it is optimal to choose reference point $\boldsymbol{c}$ as mean values of the input variables ( $\mathrm{Li}$ et al 2001b). The expansion functions are determined by evaluating the input-output responses of the system relative to the defined reference point $\boldsymbol{c}$ along associated lines, surfaces, subvolumes, etc. (i.e., cuts) in the input variable space. This process reduces to the following relationship for the component functions in Equation 2

$$
\begin{gathered}
g_{0}=g(\boldsymbol{c}), \\
g_{i}\left(x_{i}\right)=g\left(x_{i}, \boldsymbol{c}^{i}\right)-g_{0},
\end{gathered}
$$




$$
g_{i_{1} i_{2}}\left(x_{i_{1}}, x_{i_{2}}\right)=g\left(x_{i_{1}}, x_{i_{2}}, c^{i_{1} i_{2}}\right)-g_{i_{1}}\left(x_{i_{1}}\right)-g_{i_{2}}\left(x_{i_{2}}\right)-g_{0},
$$

where the notation $g\left(x_{i}, c^{i}\right)=g\left(c_{1}, c_{2}, \ldots, c_{i-1}, x_{i}, c_{i+1}, \ldots, c_{N}\right)$ denotes that all the input variables are at their reference point values except $x_{i}$. The $g_{0}$ term is the output response of the system evaluated at the reference point $c$. The higher order terms are evaluated as cuts in the input variable space through the reference point. Therefore, each first-order term $g_{i}\left(x_{i}\right)$ is evaluated along its variable axis through the reference point. Each second-order term $g_{i_{1} i_{2}}\left(x_{i_{1}}, x_{i_{2}}\right)$ is evaluated in a plane defined by the binary set of input variables $x_{i_{1}}, x_{i_{2}}$ through the reference point, etc. The process of subtracting off the lower order expansion functions removes their dependence to assure a unique contribution from the new expansion function.

Considering the terms up to first- and second-order in Equation 2 yields, respectively

$$
g(\boldsymbol{x})=g_{0}+\sum_{i=1}^{N} g_{i}\left(x_{i}\right)+\mathcal{R}_{2}
$$

and

$$
g(\boldsymbol{x})=g_{0}+\sum_{i=1}^{N} g_{i}\left(x_{i}\right)+\sum_{1 \leq i_{1}<i_{2} \leq N} g_{i_{1} i_{2}}\left(x_{i_{1}}, x_{i_{2}}\right)+\mathcal{R}_{3} .
$$

Substituting Equations 3-5 into Equations 6 and 7 leads to

$$
g(\boldsymbol{x})=\sum_{i=1}^{N} g\left(c_{1}, \ldots, c_{i-1}, x_{i}, c_{i+1}, \ldots, c_{N}\right)-(N-1) g(\boldsymbol{c})+\mathcal{R}_{2},
$$

and

$$
\begin{aligned}
g(\boldsymbol{x})= & \sum_{\substack{i_{1}=1, i_{2}=1 \\
i_{1}<i_{2}}}^{N} g\left(c_{1}, \ldots, c_{i_{1}-1}, x_{i_{1}}, c_{i_{1}+1}, \ldots, c_{i_{2}-1}, x_{i_{2}}, c_{i_{2}+1}, \ldots, c_{N}\right) \\
& -(N-2) \sum_{i=1}^{N} g\left(c_{1}, \ldots, c_{i-1}, x_{i}, c_{i+1}, \ldots, c_{N}\right) \\
& +\frac{(N-1)(N-2)}{2} g(\boldsymbol{c})+\mathcal{R}_{3},
\end{aligned}
$$

respectively. Now consider first- and second-order approximation of $\tilde{g}(\boldsymbol{x})$, denoted respectively by

$$
\tilde{g}(\boldsymbol{x})=\sum_{i=1}^{N} g\left(c_{1}, \ldots, c_{i-1}, x_{i}, c_{i+1}, \ldots, c_{N}\right)-(N-1) g(\boldsymbol{c}),
$$

and

$$
\begin{aligned}
\tilde{g}(\boldsymbol{x})= & \sum_{\substack{i_{1}=1, i_{2}=1 \\
i_{1}<i_{2}}}^{N} g\left(c_{1}, \ldots, c_{i_{1}-1}, x_{i_{1}}, c_{i_{1}+1}, \ldots, c_{i_{2}-1}, x_{i_{2}}, c_{i_{2}+1}, \ldots, c_{N}\right) \\
& -(N-2) \sum_{i=1}^{N} g\left(c_{1}, \ldots, c_{i-1}, x_{i}, c_{i+1}, \ldots, c_{N}\right)+\frac{(N-1)(N-2)}{2} g(\boldsymbol{c}) .
\end{aligned}
$$


Comparison of Equations 8 and 10 indicates that the first-order approximation leads to the residual error $g(\boldsymbol{x})-\tilde{g}(\boldsymbol{x})=R_{2}$, which includes contributions from terms of second and higher order component functions. Similarly, the second-order approximation leads to the residual error $g(\boldsymbol{x})-\tilde{g}(\boldsymbol{x})=R_{3}$, which includes contributions from terms of third and higher order component functions.

The notion of $0^{\text {th }}, 1^{\text {st }}, 2^{\text {nd }}$ order, etc. in the HDMR expansion should not be confused with the terminology used either in the Taylor series or in the conventional polynomial based approximation formulas. It can be shown that, the first-order component function $g_{i}\left(x_{i}\right)$ is the sum of all the Taylor series terms which contain and only contain variable $x_{i}$. Similarly, the second-order component function $g_{i_{1} i_{2}}\left(x_{i_{1}}, x_{i_{2}}\right)$ is the sum of all the Taylor series terms which contain and only contain variables $x_{i_{1}}$ and $x_{i_{2}}$. Hence first- and second-order HDMR approximations should not be viewed as first- or second-order Taylor series expansions nor do they limit the nonlinearity of $g(\boldsymbol{x})$. Furthermore, the approximations contain contributions from all input variables. Thus, the infinite number of terms in the Taylor series is partitioned into finite different groups and each group corresponds to one cut-HDMR component function. Therefore, any truncated cut-HDMR expansion provides a better approximation and convergent solution of $g(\boldsymbol{x})$ than any truncated Taylor series because the latter contains only a finite number of terms of Taylor series. Furthermore, the coefficients associated with higher dimensional terms are usually much smaller than that with one-dimensional terms. As such, the impact of higher dimensional terms on the function is less, and therefore, can be neglected. Compared with the first- and second-order Taylor series expansions, which retain only linear and quadratic terms, respectively, the first-order HDMR provides more accurate approximation $\tilde{g}(\boldsymbol{x})$ of the original response function $g(\boldsymbol{x})$. If the first-order HDMR approximation is not sufficient, the second-order HDMR approximation may be adopted at the expense of additional computational cost.

\section{HDMR based response surface generation}

HDMR in Equation 2 is exact along any of the cuts, and the output response $g(\boldsymbol{x})$ at a point $\boldsymbol{x}$ off of the cuts can be obtained as follows.

Step 1: Interpolate each of the low dimensional HDMR expansion terms with respect to the input values of the point $\boldsymbol{x}$. For example, consider the first-order component function $g\left(x_{i}, c^{i}\right)=g\left(c_{1}, c_{2}, \ldots, c_{i-1}, x_{i}, c_{i+1}, \ldots, c_{N}\right)$. If for $x_{i}=x_{i}^{j}, n$ function values

$$
g\left(x_{i}^{j}, \boldsymbol{c}^{i}\right)=g\left(c_{1}, \ldots, c_{i-1}, x_{i}^{j}, c_{i+1}, \ldots, c_{N}\right) ; j=1,2, \ldots, n,
$$

are given at $n(=3,5,7$ or 9$)$ sample points $x_{i L}, x_{i M}-(n-3)\left(x_{i M}-x_{i L}\right) /(n-1), x_{i M}-$ $(n-5)\left(x_{i M}-x_{i L}\right) /(n-1), \ldots, \quad x_{i M}, \ldots, x_{i M}+(n-5)\left(x_{i U}-x_{i M}\right) /(n-1), x_{i M}+$ $(n-3)\left(x_{i U}-x_{i M}\right) /(n-1), x_{i U}$, along the fuzzy variable $x_{i}$ having triangular membership function with the triplet number $\left[x_{i L}, x_{i M}, x_{i U}\right]$. The function value for arbitrary $x_{i}$ can be obtained by the Lagrange interpolation as

$$
g\left(x_{i}, c^{i}\right)=\sum_{j=1}^{n} \phi_{j}\left(x_{i}\right) g\left(c_{1}, \ldots, c_{i-1}, x_{i}^{j}, c_{i+1}, \ldots, c_{N}\right) ; j=1,2, \ldots, n
$$


Similarly, consider the second-order component function $g\left(x_{i_{1}}, x_{i_{2}}, \boldsymbol{c}^{i_{1} i_{2}}\right)=g\left(c_{1}, \ldots, c_{i_{1}-1}\right.$, $\left.x_{i_{1}}, c_{i_{1}+1}, \ldots, c_{i_{2}-1}, x_{i_{2}}, c_{i_{2}+1}, \ldots, c_{N}\right)$. If for $x_{i_{1}}=x_{i_{1}}^{j_{1}}$, and $x_{i_{2}}=x_{i_{2}}^{j_{2}}, n^{2}$ function values

$$
\begin{gathered}
g\left(x_{i_{1}}^{j_{1}}, x_{i_{2}}^{j_{2}}, c^{i_{1} i_{2}}\right)=g\left(c_{1}, \ldots, c_{i_{1}-1}, x_{i_{1}}^{j_{1}}, c_{i_{1}+1}, \ldots, c_{i_{2}-1}, x_{i_{2}}^{j_{2}}, c_{i_{2}+1}, \ldots, c_{N}\right) \\
j_{1}=1,2, \ldots, n, \quad j_{2}=1,2, \ldots, n
\end{gathered}
$$

are given on a grid formed by taking $n(=3,5,7$ or 9$)$ sample points along $x_{i_{1}}$ and $x_{i_{2}}$ axes as explained above, then the function value for arbitrary $\left(x_{i_{1}}, x_{i_{2}}\right)$ can be obtained by the Lagrange interpolation as

$$
\begin{aligned}
g\left(x_{i_{1}}, x_{i_{2}}, \boldsymbol{c}^{i_{1} i_{2}}\right)= & \sum_{j_{1}=1}^{n} \sum_{j_{2}=1}^{n} \phi_{j_{1} j_{2}}\left(x_{i_{1}}, x_{i_{2}}\right) \\
& g\left(c_{1}, \ldots, c_{i_{1}-1}, x_{i_{1}}^{j_{1}}, c_{i_{1}+1}, \ldots, c_{i_{2}-1}, x_{i_{2}}^{j_{2}}, c_{i_{2}+1}, \ldots, c_{N}\right),
\end{aligned}
$$

where the interpolation functions $\phi_{j}\left(x_{i}\right)$ and $\phi_{j_{1} j_{2}}\left(x_{i_{1}}, x_{i_{2}}\right)$ can be obtained using the Lagrange interpolation.

By using Equation 13, $g_{i}\left(x_{i}\right)$ can be generated if $n$ function values are given at corresponding sample points. Similarly, by using Equation 15, $g_{i_{1} i_{2}}\left(x_{i_{1}}, x_{i_{2}}\right)$ can be generated if $n^{2}$ function values at corresponding sample points are given. The same procedure shall be repeated for all the first-order component functions, i.e., $g_{i}\left(x_{i}\right) ; i=1,2, \ldots, N$ and the second-order component functions, i.e., $g_{i_{1} i_{2}}\left(x_{i_{1}}, x_{i_{2}}\right) ; i_{1}, i_{2}=1,2, \ldots, N$.

Step 2: Sum the interpolated values of HDMR expansion terms from zeroth-order to the highest order retained in keeping with the desired accuracy. This leads to first-order approximation of the function $g(\boldsymbol{x})$ as

$$
\tilde{g}(\boldsymbol{x})=\sum_{i=1}^{N} \sum_{j=1}^{n} \phi_{j}\left(x_{i}\right) g\left(c_{1}, \ldots, c_{i-1}, x_{i}^{j}, c_{i+1}, \ldots, c_{N}\right)-(N-1) g_{0},
$$

and second-order approximation of the function $g(\boldsymbol{x})$ as

$$
\begin{aligned}
\tilde{g}(\boldsymbol{x})= & \sum_{\substack{i_{1}=1, i_{2}=1 \\
i_{1}<i_{2}}}^{N} \sum_{j_{1}=1}^{n} \sum_{j_{2}=1}^{n} \phi_{j_{1} j_{2}}\left(x_{i_{1}}, x_{i_{2}}\right) \\
& g\left(c_{1}, \ldots, c_{i_{1}-1}, x_{i_{1}}^{j_{1}}, c_{i_{1}+1}, \ldots, c_{i_{2}-1}, x_{i_{2}}^{j_{2}}, c_{i_{2}+1}, \ldots, c_{N}\right) \\
& -(N-2) \sum_{i=1}^{N} \sum_{j=1}^{n} \phi_{j}\left(x_{i}\right) g\left(c_{1}, \ldots, c_{i-1}, x_{i}^{j}, c_{i+1}, \ldots, c_{N}\right)+\frac{(N-1)(N-2)}{2} g_{0} .
\end{aligned}
$$

The interpolation functions $\phi_{j}\left(x_{i}\right)$ and $\phi_{j_{1} j_{2}}\left(x_{i_{1}}, x_{i_{2}}\right)$ by using the Lagrange interpolation are defined as

$$
\phi_{j}\left(x_{i}\right)=\frac{\left(x_{i}-x_{i}^{(1)}\right) \cdots\left(x_{i}-x_{i}^{(j-1)}\right)\left(x_{i}-x_{i}^{(j+1)}\right) \cdots\left(x_{i}-x_{i}^{(n)}\right)}{\left(x_{i}^{(j)}-x_{i}^{(1)}\right) \cdots\left(x_{i}^{(j)}-x_{i}^{(j-1)}\right)\left(x_{i}^{(j)}-x_{i}^{(j+1)}\right) \cdots\left(x_{i}^{(j)}-x_{i}^{(n)}\right)},
$$




$$
\begin{aligned}
& \phi_{j_{1} j_{2}}\left(x_{i_{1}}, x_{i_{2}}\right) \\
& =\left[\frac{\left(x_{i_{1}}-x_{i_{1}}^{(1)}\right) \cdots\left(x_{i_{1}}-x_{i_{1}}^{\left(j_{1}-1\right)}\right)\left(x_{i_{1}}-x_{i_{1}}^{\left(j_{1}+1\right)}\right) \cdots\left(x_{i_{1}}-x_{i_{1}}^{(n)}\right)}{\left(x_{i_{1}}^{\left(j_{1}\right)}-x_{i_{1}}^{(1)}\right) \cdots\left(x_{i_{1}}^{\left(j_{1}\right)}-x_{i_{1}}^{\left(j_{1}-1\right)}\right)\left(x_{i_{1}}^{\left(j_{1}\right)}-x_{i_{1}}^{\left(j_{1}+1\right)}\right) \cdots\left(x_{i_{1}}^{\left(j_{1}\right)}-x_{i_{1}}^{(n)}\right)}\right] \\
& \times\left[\frac{\left(x_{i_{2}}-x_{i_{2}}^{(1)}\right) \cdots\left(x_{i_{2}}-x_{i_{2}}^{\left(j_{2}-1\right)}\right)\left(x_{i_{2}}-x_{i_{2}}^{\left(j_{2}+1\right)}\right) \cdots\left(x_{i_{2}}-x_{i_{2}}^{(n)}\right)}{\left(x_{i_{2}}^{\left(j_{2}\right)}-x_{i_{2}}^{(1)}\right) \cdots\left(x_{i_{2}}^{\left(j_{2}\right)}-x_{i_{2}}^{\left(j_{2}-1\right)}\right)\left(x_{i_{2}}^{\left(j_{2}\right)}-x_{i_{2}}^{\left(j_{2}+1\right)}\right) \cdots\left(x_{i_{2}}^{\left(j_{2}\right)}-x_{i_{2}}^{(n)}\right)}\right] .
\end{aligned}
$$

If $n$ is the number of sample points taken along each of the variable axis and $s$ is the order of the component function considered, starting from zeroth-order to $l$-th order, then total number of function evaluation for interpolation purpose is given by, $\sum_{s=0}^{l}\left(N !(n-1)^{s}\right) /((N-s) ! s !)$ which grows polynomially with $N$ and $n$, and exponentially with $s$. As a few low order component functions of HDMR are used, the sample savings due to HDMR are significant compared to traditional sampling. Hence uncertainty analysis using HDMR relies on an accurate reduced model being generated with a small number of full model simulations. The tremendous computational savings result from just having to perform interpolation instead of full model simulations for output determination.

\section{Explicit fuzzy analysis}

The original response function $g(\boldsymbol{x})$ is approximated using HDMR concepts presented in sections 3 and 4 . Once the response function is expressed as a linear combination of the fuzzy variables, the bounds of the response at each $\alpha$-cut can be obtained by using interval arithmetic algorithm. In order to apply interval arithmetic algorithm, the first-order HDMR approximation $\tilde{g}(\boldsymbol{x})$ of the original nonlinear response function $g(\boldsymbol{x})$ is transformed into a linear combination of intervening variables as follows.

$$
\tilde{g}(z)=z_{1}+z_{2}+\ldots+z_{N}+\beta_{0} .
$$

where $z_{i}=\left(\beta_{i} x_{i}+\gamma_{i}\right)^{\kappa}$ is the relation between the intervening and the original variables with $\kappa$ being order of the first-order HDMR component functions approximation taking values $\kappa=$ 1 for linear approximation, $\kappa=2$ for quadratic approximation, $\kappa=3$ for cubic approximation, and so on. If the membership functions of the intervening variables are available, then at each $\alpha$ cut, interval arithmetic techniques (Moore 1979) can be applied to estimate the response bounds at that level.

Let the intervening variable, $z$, be a function of $x$ given by, $z=G(x)$. The membership function of $z$ is obtained by using the membership function of $x$. The membership function gives the possibility of occurrence. For this reason, the possibility of occurrence of a point $z_{0}$ is equal to the possibility of occurrence of $x_{0}$, where $x_{0}=G^{-1}\left(z_{0}\right)$. Therefore, the possibility function of $z$ can be obtained using the following equation and appropriate transformations is shown in figure 1:

$$
\mu_{z}(z)=\mu_{x}\left(g^{-1}(z)\right)
$$

The membership function of $z$ can be obtained numerically by the use of transformations.

Once the first-order HDMR approximation $\tilde{g}(\boldsymbol{x})$ of the original nonlinear response function $g(\boldsymbol{x})$ is expressed as a linear combination in terms of intervening variables (Equation 20), interval 


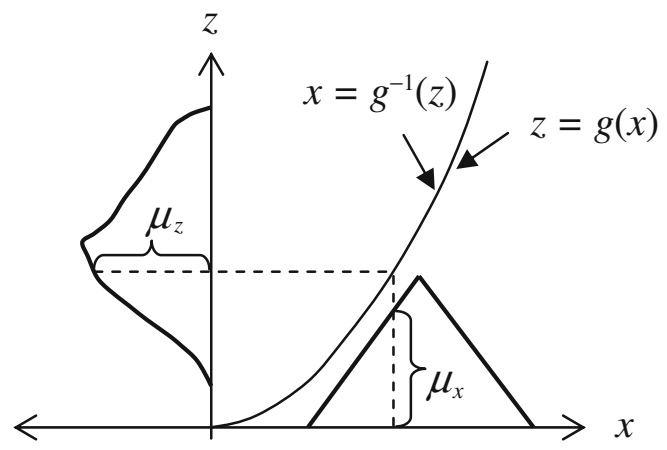

Figure 1. Transformation to obtain membership function of intervening variable.

arithmetic techniques (Moore 1979; Alefeld \& Herzberger 1983) can be used to estimate the response bounds. The drawback of using interval arithmetic (Hansen 1992) for a closed-form expression is the problem with dependency. In general, if any variable appears more than once in an expression, it is treated as a different variable for each occurrence, resulting in wider bounds. By using intervening variables, each variable appears only once in the expression, resulting in accurate bounds on the response.

Similar transformation techniques are adopted by Adduri \& Penmetsa (2008) to obtain minimum and maximum values of a nonlinear response at each $\alpha$-cut, except that a second-order response surface model is used for the original response approximation, however the proposed first-order HDMR provides better approximation $\tilde{g}(\boldsymbol{x})$ of the original response function $g(\boldsymbol{x})$.

The use of transformation techniques facilitates the determination of the minimum and maximum values of the fuzzy variables that correspond to the extreme values of the response at a particular level without the use of optimization techniques. Moreover, this is an analytical procedure in which there is little room for errors. In all the numerical examples presented in this paper, first-order HDMR approximation is adopted. A flow diagram for explicit fuzzy analysis using HDMR approximation is shown in figure 2.

\section{Numerical examples}

Four numerical examples are presented to demonstrate the accuracy and efficiency of the proposed methodology. The proposed explicit formulations can be applied to fuzzy variables with any type of membership functions to result in an accurate estimation of the bounds of the response.

If $N, n$, and $m$ respectively denote the number of fuzzy variables, the number of sample points taken along each of the variable axis, and the number of $\alpha$-cuts, then using first-order HDMR approximation the total cost of original function evaluation entails a maximum of $N \times(n-1)+1$ by the proposed explicit formulation. It can be easily verified that the total computational cost of the proposed formulations is far less than $2^{N} m+1$ number of function evaluations required for the crude vertex method fuzzy analysis in conjunction with combinatorial optimization (in which all possible combinations of the fuzzy variables are considered), especially for large number of fuzzy input parameters and large finite element models. This clearly demonstrates the computational efficiency of the proposed formulations. The efficiency and robustness of the proposed formulation is expected to increase with increase in the complexity of the structure, 


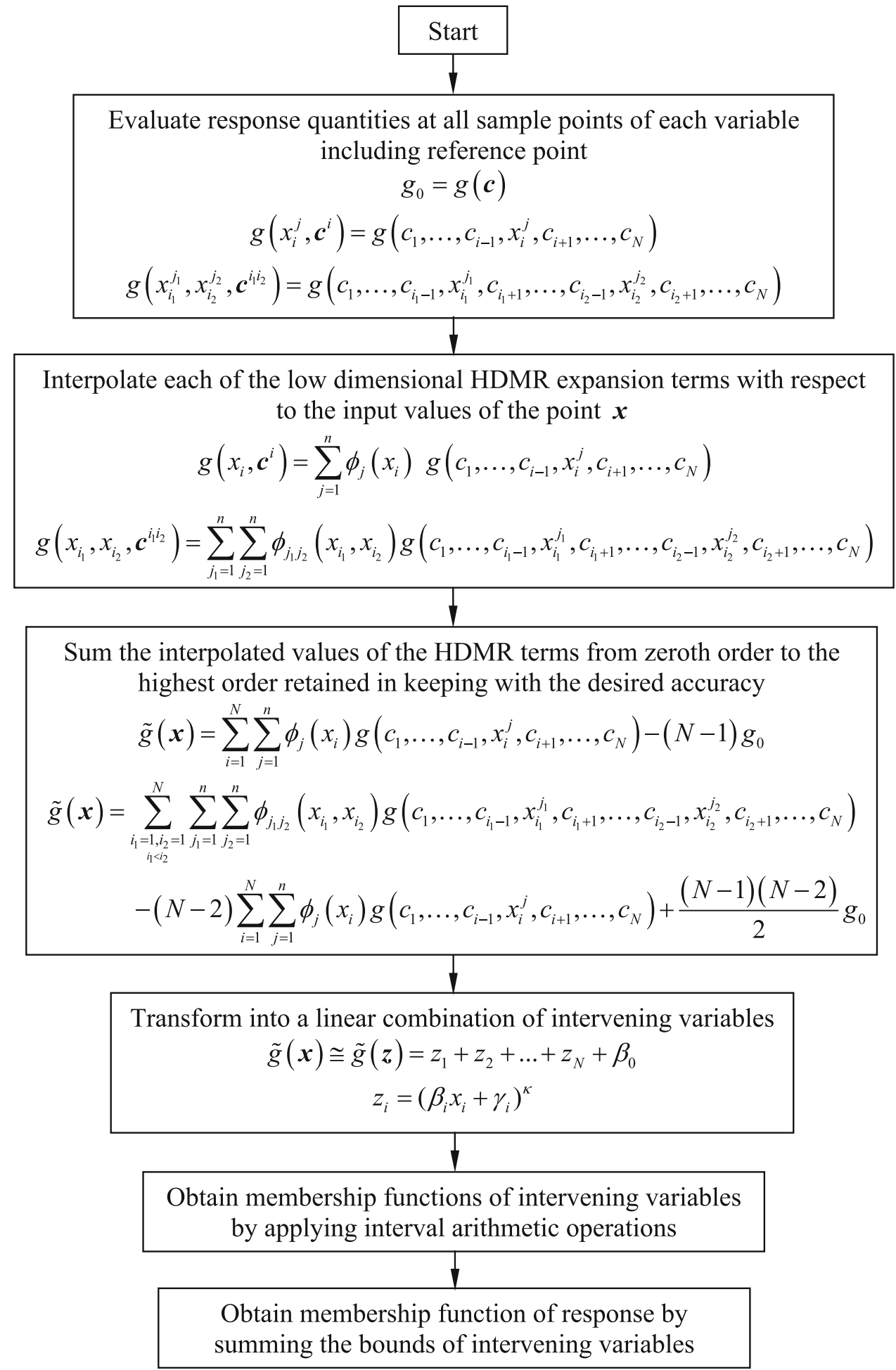

Figure 2. Flowchart of explicit fuzzy analysis using HDMR approximation. 
number of fuzzy variables and number of $\alpha$-cuts. If first-order HDMR approximation is not sufficient second-order HDMR approximation may be adopted at the expense of additional computational cost. In that case, the total cost of original function evaluation entails a maximum of $(n-1)^{2}(N-1) N / 2+(n-1) N+1$ by the proposed explicit formulation.

\subsection{Closed form example}

To illustrate the accuracy and the applicability of the transformation technique in the proposed explicit formulation to obtain the bounds on the response, the following nonlinear response function is considered.

$$
\begin{aligned}
f(\boldsymbol{x})= & 16 x_{1}^{4}-96 x_{1}^{3}+216 x_{1}^{2}-216 x_{1}-64 x_{2}^{3}+240 x_{2}^{2}-300 x_{2} \\
& +36 x_{3}^{2}-84 x_{3}-8 x_{4}+264,
\end{aligned}
$$

where $x_{1}=[1,2,3], x_{2}=[-1,0,1], x_{3}=[-5,0,5]$, and $x_{4}=[10,15,20]$ are assumed to have triangular membership functions and the triplet number for each fuzzy variable $\left[x_{L}, x_{M}, x_{U}\right]$ defines a triangular membership function, where $x_{L}$ and $x_{U}$ are the lower and upper bounds at $\alpha=0$ and $x_{M}$ is the nominal value at $\alpha=1$. As Equation 22 represents closed form nonlinear function, the HDMR approximation of the original response function is skipped and suitable transformations of variables $x_{1}-x_{4}$ are applied to form in a linear combination of intervening variables, as follows

$$
f(z)=z_{1}+z_{2}+z_{3}+z_{4}
$$

where $z_{1}=\left(2 x_{1}-3\right)^{4}, z_{2}=-\left(4 x_{2}-5\right)^{3}, z_{3}=\left(6 x_{3}-7\right)^{2}$ and $z_{4}=-8 x_{4}+9$.

Once the membership functions of the intervening variables are obtained, the interval arithmetic techniques are applied on Equation 23 to estimate the response bounds at each $\alpha$-cut to get the membership function of $f(\boldsymbol{x})$. Figure 3 shows the membership function of $f(\boldsymbol{x})$ obtained by the crude vertex method fuzzy analysis in conjunction with combinatorial optimization, by adopting interval arithmetic techniques directly on Equation 22, and by the proposed explicit formulation. As expected due to the problem of dependency the membership function of $f(\boldsymbol{x})$ obtained by directly adopting interval arithmetic techniques resulted in wider bounds. The differences in the joint membership function of $f(\boldsymbol{x})$ obtained by the crude vertex method fuzzy

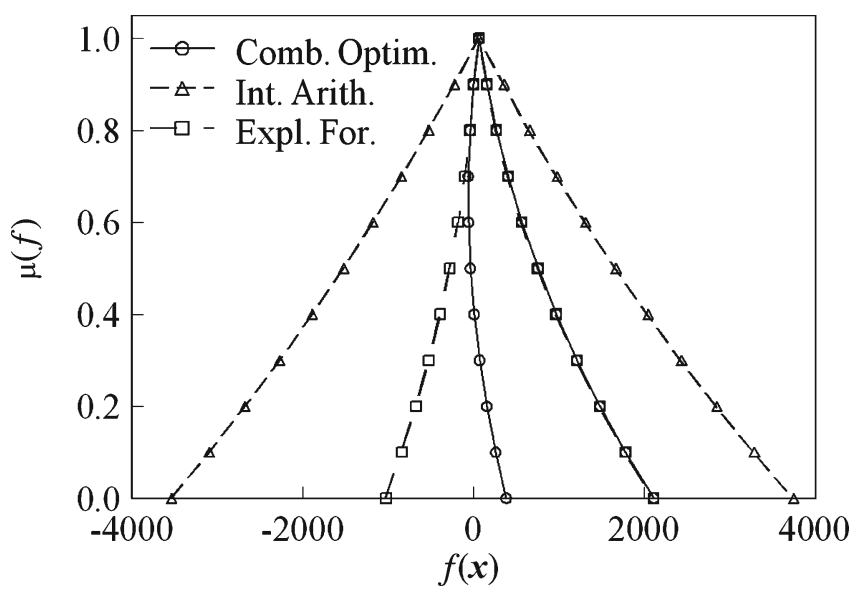

Figure 3. Comparison of membership function of response (example 1). 


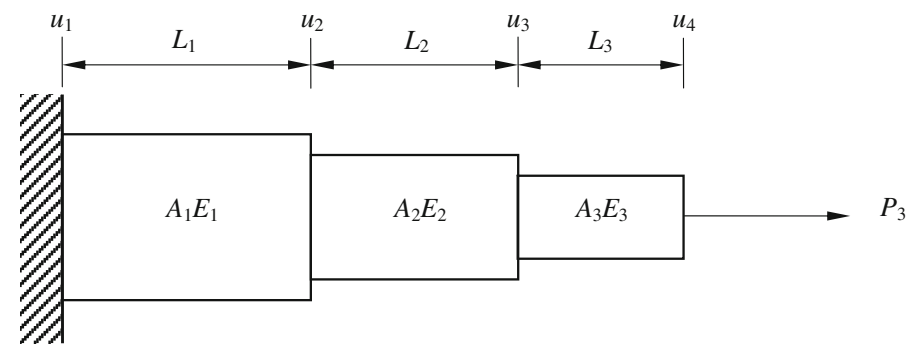

Figure 4. Three-stepped bar configuration.

analysis in conjunction with combinatorial optimization and the proposed explicit formulation is mainly due to the reason that the membership function obtained by using the vertex method fails to capture the minimum value of the response because it calculates the minimum and maximum values of the response only at the lower and upper limits of the variables at each $\alpha$-level. Therefore, it does not identify the minimum of the response, which is located inside the design space.

\subsection{Three steeped bar}

In this example, the static and dynamic responses of a three stepped bar (Akpan et al 2001a) shown in figure 4 , are considered.

6.2a Static analysis: The following three cases of fuzzy input variables are considered for the static analysis: (i) Case A in which only the load $P$ is fuzzified; (ii) Case B in which both the

Table 1. Geometrical and material properties of three-stepped bar (example 2).

\begin{tabular}{lccc}
\hline Parameter & Case A & Case B & Case C \\
\hline & \multicolumn{1}{c}{ Data for fuzzy static analysis of three stepped bar } & \\
$A_{1}$ (in. $\left.^{2}\right)$ & {$[3.00,3.00,3.00]$} & {$[3.00,3.00,3.00]$} & {$[2.99,3.00,3.01]$} \\
$A_{2}$ (in. $\left.^{2}\right)$ & {$[2.00,2.00,2.00]$} & {$[3.00,3.00,3.00]$} & {$[1.99,2.00,2.01]$} \\
$A_{3}$ (in. $\left.^{2}\right)$ & {$[1.00,1.00,1.00]$} & {$[1.00,1.00,1.00]$} & {$[0.99,1.00,1.01]$} \\
$L_{1}$ (in.) & {$[12.00,12.00,12.00]$} & {$[12.00,12.00,12.00]$} & {$[11.95,12.00,12.05]$} \\
$L_{2}$ (in.) & {$[10.00,10.00,10.00]$} & {$[10.00,10.00,10.00]$} & {$[9.95,10.00,10.05]$} \\
$L_{3}$ (in.) & {$[6.00,6.00,6.00]$} & {$[6.00,6.00,6.00]$} & {$[5.95,6.00,6.05]$} \\
$E_{1}, E_{2}, E_{3}$ (psi) & {$[3.0 \mathrm{e} 7,3.0 \mathrm{e} 7,3.0 \mathrm{e} 7]$} & {$[2.8 \mathrm{e} 7,3.0 \mathrm{e} 7,3.1 \mathrm{e} 7]$} & {$[2.8 \mathrm{e} 7,3.0 \mathrm{e} 7,3.1 \mathrm{e} 7]$} \\
$P_{1}, P_{2}(\mathrm{lb})$ & {$[0.0,0.0,0.0]$} & {$[0.0,0.0,0.0]$} & {$[0.0,0.0,0.0]$} \\
$P_{3}$ (lb) & {$[7500,10000,12500]$} & {$[7500,10000,12500]$} & {$[7500,10000,12500]$} \\
& & & \\
$A_{1}$ (in. $\left.{ }^{2}\right)$ & Data for fuzzy dynamic analysis of three stepped bar & \\
$A_{2}$ (in. $\left.{ }^{2}\right)$ & {$[3.00,3.00,3.00]$} & {$[3.00,3.00,3.00]$} & {$[2.99,3.00,3.01]$} \\
$A_{3}$ (in. $\left.{ }^{2}\right)$ & {$[2.00,2.00,2.00]$} & {$[3.00,3.00,3.00]$} & {$[1.99,2.00,2.01]$} \\
$L_{1}$ (in.) & {$[1.00,1.00,1.00]$} & {$[1.00,1.00,1.00]$} & {$[0.99,1.00,1.01]$} \\
$L_{2}$ (in.) & {$[12.00,12.00,12.00]$} & {$[12.00,12.00,12.00]$} & {$[11.95,12.00,12.05]$} \\
$L_{3}$ (in.) & {$[10.00,10.00,10.00]$} & {$[10.00,10.00,10.00]$} & {$[9.95,10.00,10.05]$} \\
$E_{1}, E_{2}, E_{3}$ (psi) & {$[6.00,6.00,6.00]$} & {$[6.00,6.00,6.00]$} & {$[5.95,6.00,6.05]$} \\
$\rho_{1}, \rho_{2}, \rho_{3}$ (lb/in. $\left.{ }^{3}\right)$ & {$[3.0 \mathrm{e} 7,3.0 \mathrm{e} 7,3.0 \mathrm{e} 7]$} & {$[2.8 \mathrm{e} 7,3.0 \mathrm{e} 7,3.1 \mathrm{e} 7]$} & {$[2.8 \mathrm{e} 7,3.0 \mathrm{e} 7,3.1 \mathrm{e} 7]$} \\
\hline
\end{tabular}


load and the elastic modulus are fuzzified; and (iii) Case $\mathrm{C}$ in which the load, elastic modulus, cross sectional areas and the lengths of the bar are taken as fuzzy variables. The details of fuzzy input variables for all three cases are presented in table 1 .

The possibility distributions of the fuzzy axial displacement at the free end and the strain energy of the system are computed using the proposed first-order HDMR based explicit formulation. The effect of number of sample points used for first-order HDMR approximation of the original response function is examined by varying $n$ from 3 to 9 . It is observed that $n=7$ results in the convergent estimation of the bounds of the response using the proposed explicit formulation. In addition, the effect of order of approximation $\kappa$ is examined by taking $\kappa=2,3$ and 4 . The possibility distributions of the fuzzy axial displacement at the free end for Case A obtained using the proposed first-order HDMR based explicit (with $n=7$ ) formulations and the crude vertex method fuzzy analysis in conjunction with combinatorial optimization are presented in figure 5(a). Similarly, figure 5(b) compares the possibility distributions of the strain energy of the system for Case A.
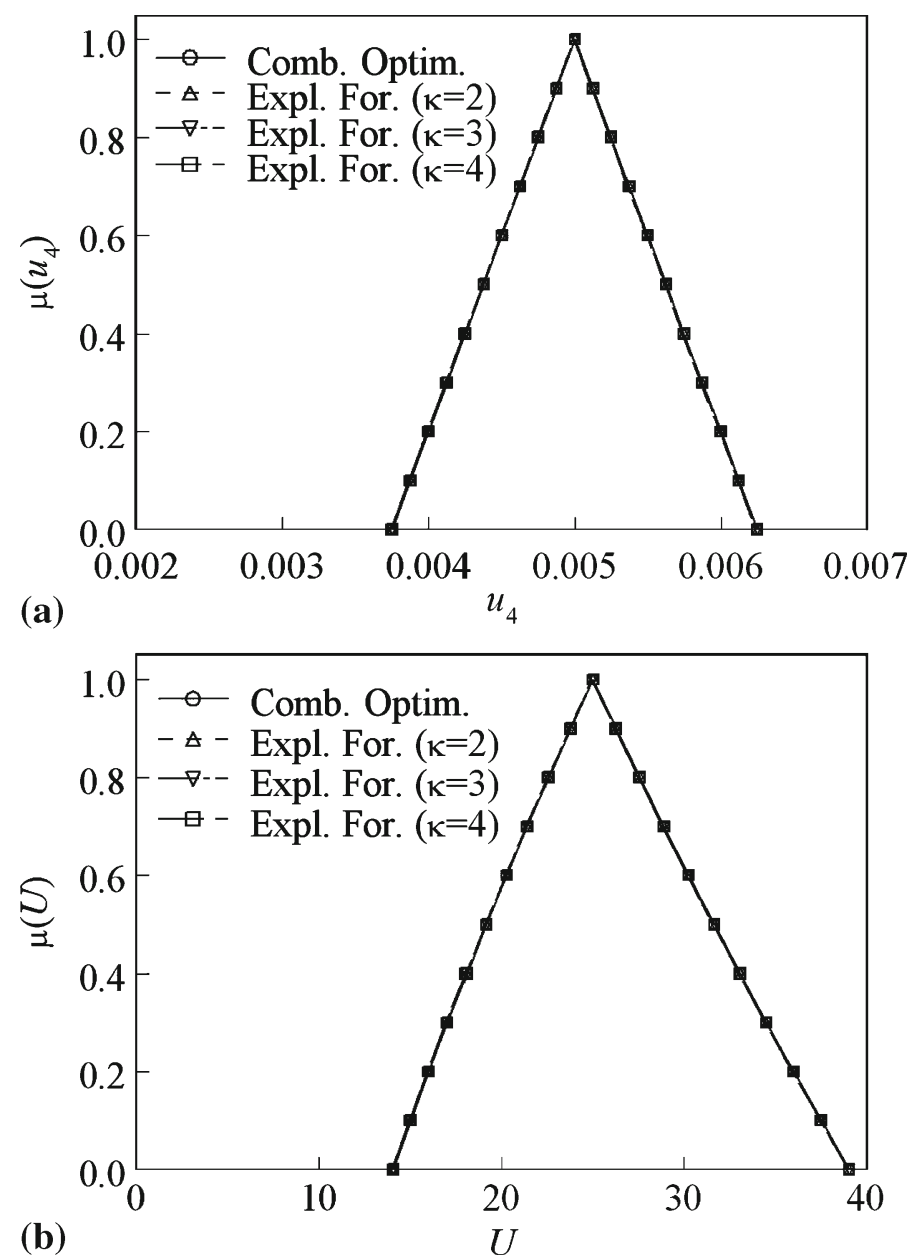

Figure 5. Possibility distributions for Case A (example 2); (a) Fuzzy axial displacement at free end; and (b) Strain energy of system. 
Figures 6(a) and (b) respectively present the possibility distributions of the fuzzy axial displacement at the free end and the strain energy of the system for Case B. Similarly, figures 7(a) and (b) respectively present the possibility distributions of the fuzzy axial displacement at the free end and the strain energy of the system for Case C. Similar to the observations reported by Akpan et al (2001a), the present study shows that as the number of fuzzy variables increases the bounds of the possibility distributions of both the displacements and the strain energy are also increased. Also it shows that for Case B and Case C the possibility distributions of the response are skewed to the right because the displacement and the strain energy are inversely proportional to the modulus of elasticity, which is skewed to the left. Since in Case A only one fuzzy variable $(P)$ is present the dependency problem is not encountered and the proposed firstorder HDMR based explicit formulation, and the combinatorial approach yield exactly the same results for all the possibility distributions of the responses. For Case $\mathrm{B}$ and Case $\mathrm{C}$ the proposed first-order HDMR based explicit formulation estimates a narrow bound for the response and with increase in the order of approximation $\kappa$, the bounds for the response are observed to become still narrower.
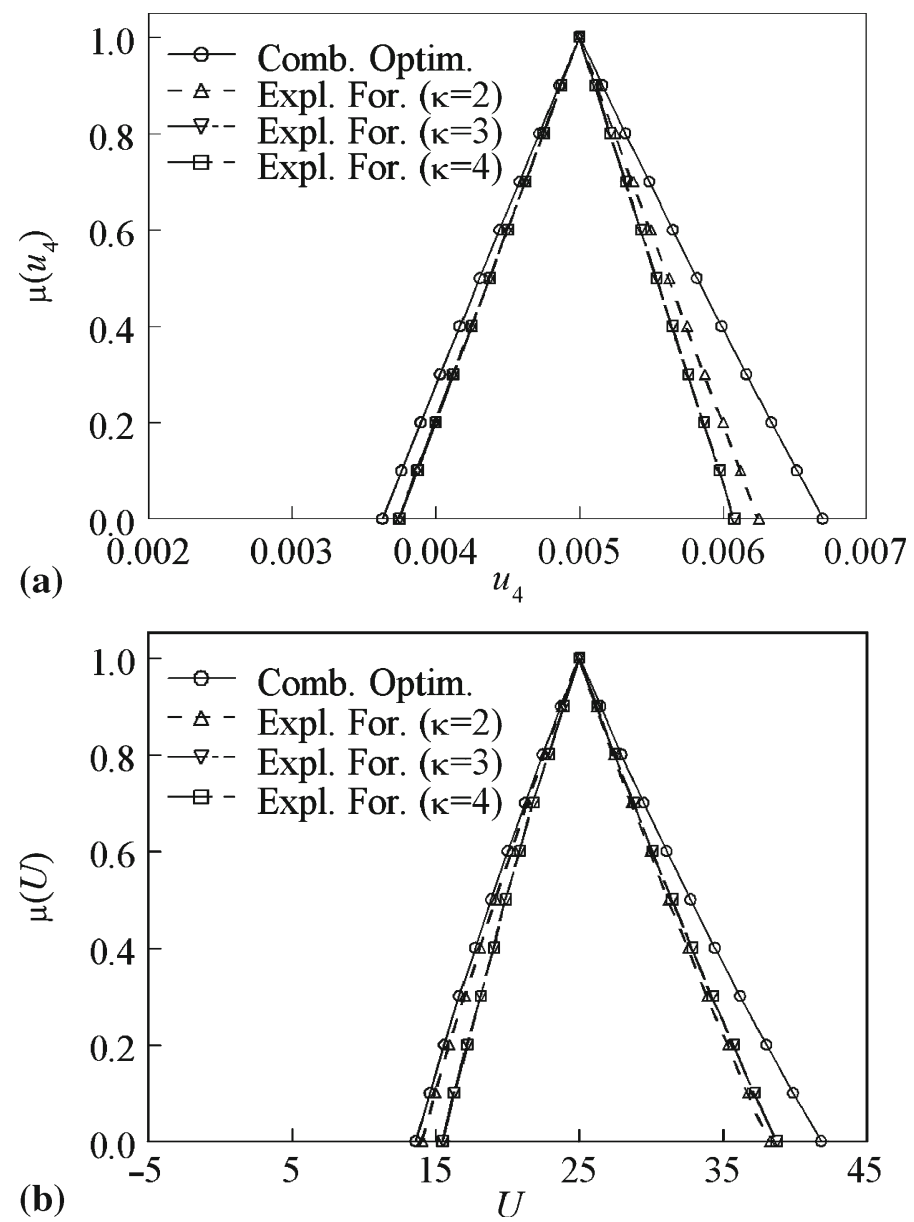

Figure 6. Possibility distributions for Case B (example 2); (a) Fuzzy axial displacement at free end; and (b) Strain energy of system. 

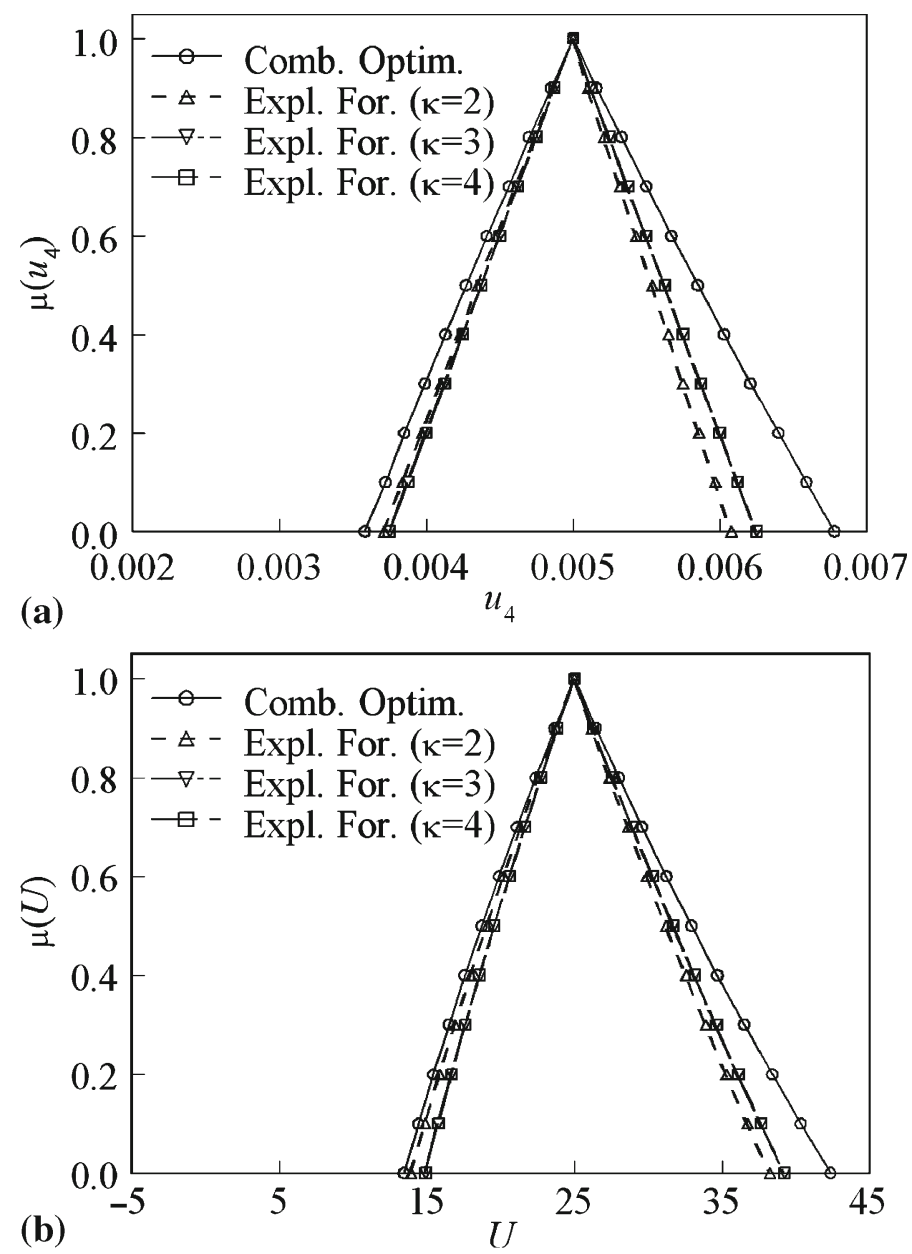

Figure 7. Possibility distributions for Case C (example 2); (a) Fuzzy axial displacement at free end; and (b) Strain energy of system.

6.2b Natural frequency analysis: The effect of input parameter fuzzification on the natural frequencies is studied by considering the following three cases: (i) Case A in which only the density of the bars as fuzzy; (ii) Case B in which both the density and the modulus of elasticity are taken as fuzzy variables; and (iii) Case $\mathrm{C}$ in which the density, modulus of elasticity, crosssectional area and length of each section are considered as fuzzy. The triangular fuzzy numbers for each parameter considered are shown in table 1.

The fuzzy natural frequencies of modes 1-3 for Case A, Case B, and Case C are respectively shown in figures 8(a)-(c), 9(a)-(c), and 10(a)-(c). Again, for Case A since only one fuzzy variable $(P)$ is present, the dependency problem is not encountered and the proposed first-order HDMR based explicit formulation, and the combinatorial approach yield exactly the same results for all the possibility distributions of the responses. For Case B and Case $\mathrm{C}$ trends for the possibility distributions of the fuzzy natural frequencies are similar to the possibility distributions of the static responses. Similar to the possibility distributions of the static responses as the number of input fuzzy variables increases from Case A to Case $C$ the spread of fuzzy frequencies also 

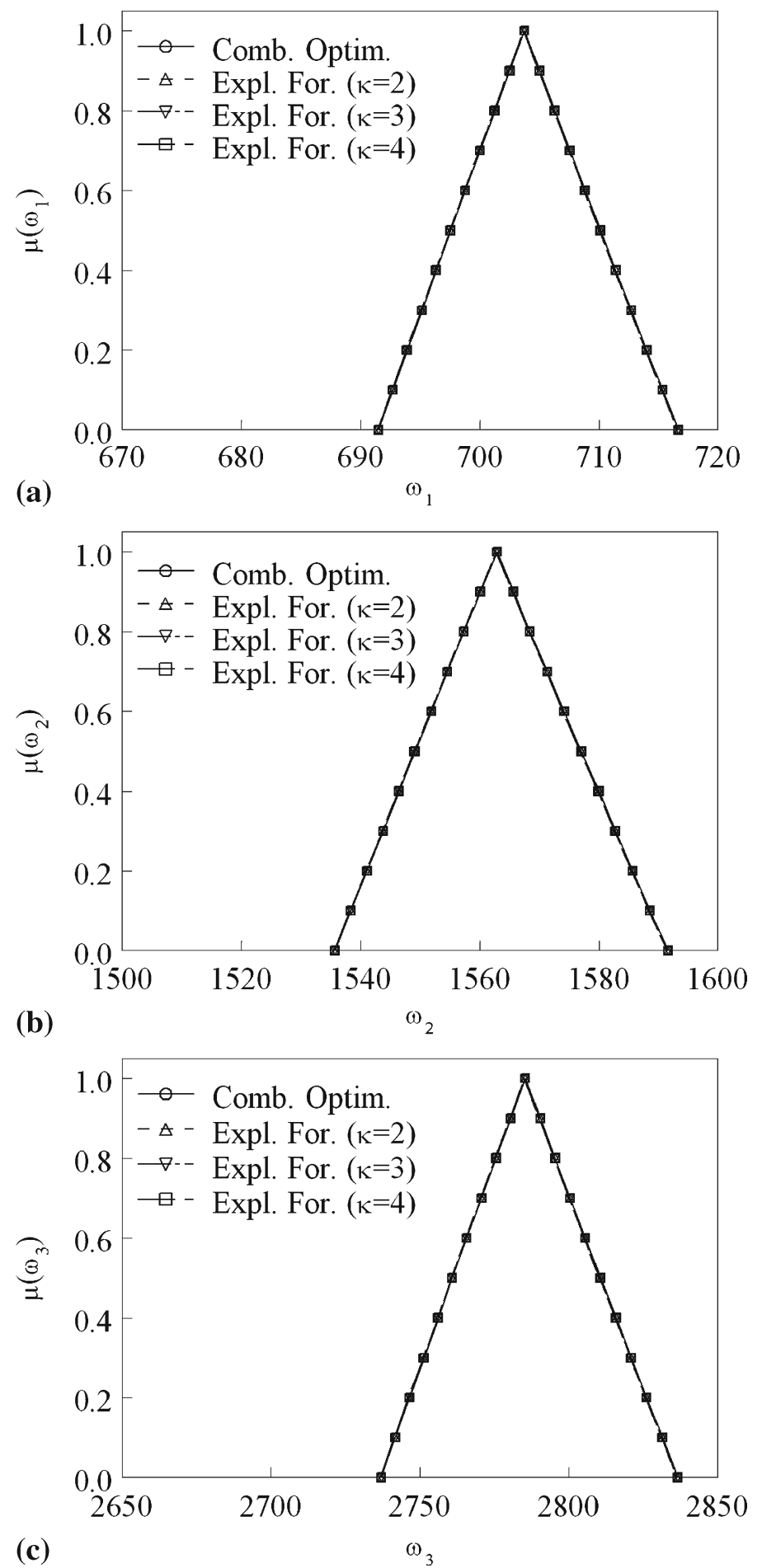

Figure 8. Possibility distributions of natural frequencies for Case A (example 2); (a) Mode 1; (b) Mode 2; and (c) Mode 3. 

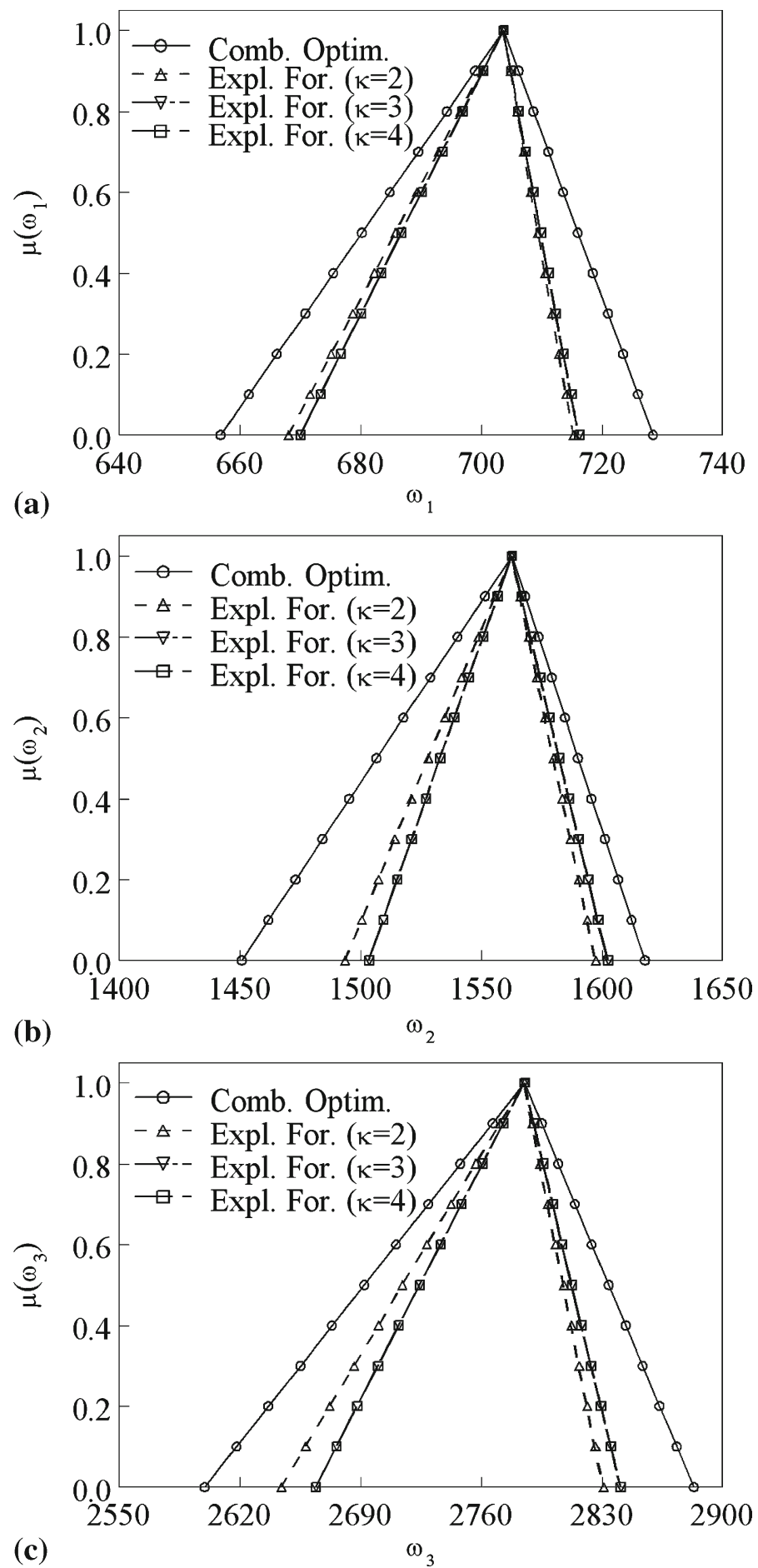

Figure 9. Possibility distributions of natural frequencies for Case B (example 2); (a) Mode 1; (b) Mode 2; and (c) Mode 3. 




(a)

$\omega_{1}$
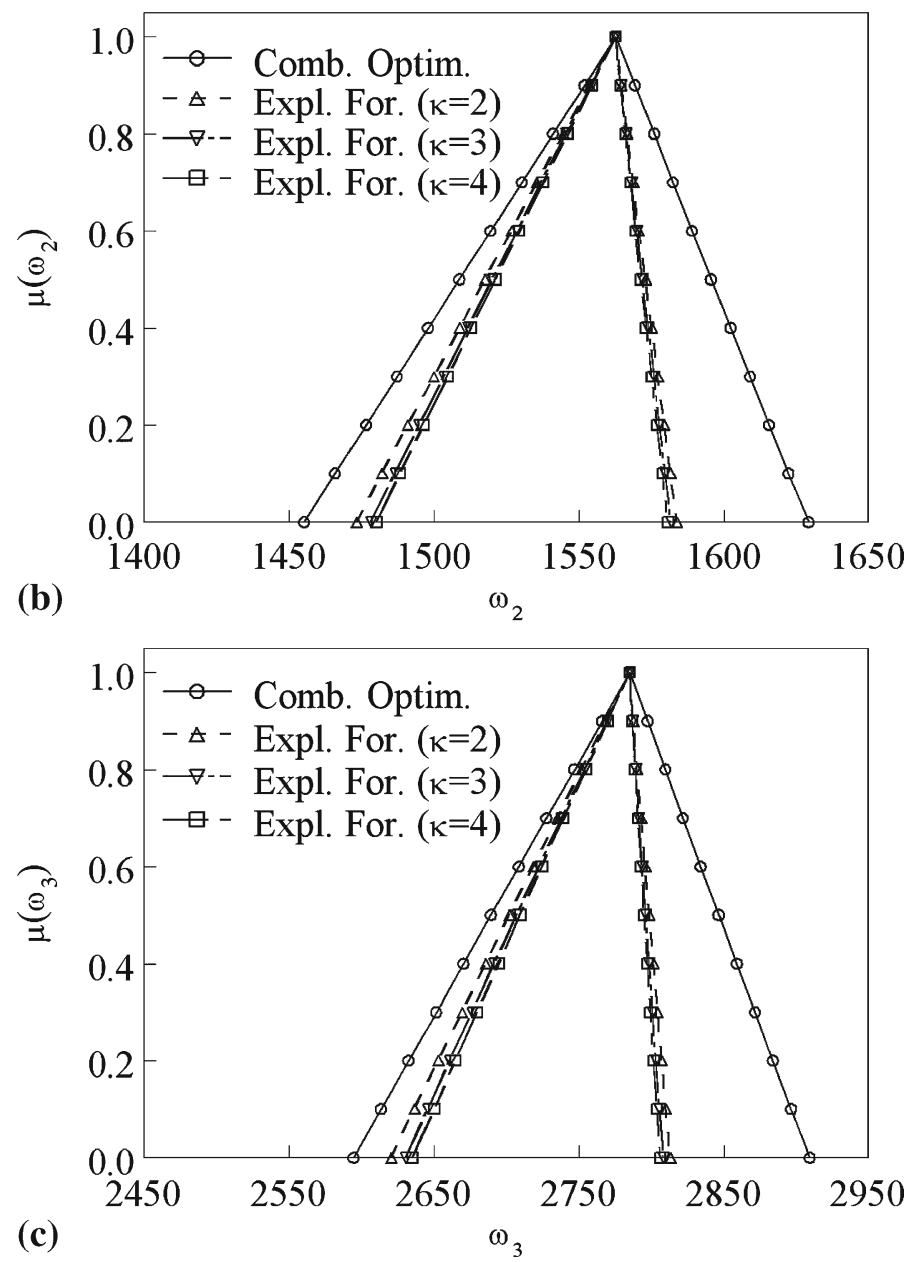

Figure 10. Possibility distributions of natural frequencies for Case C (example 2); (a) Mode 1; (b) Mode 2; and (c) Mode 3. 


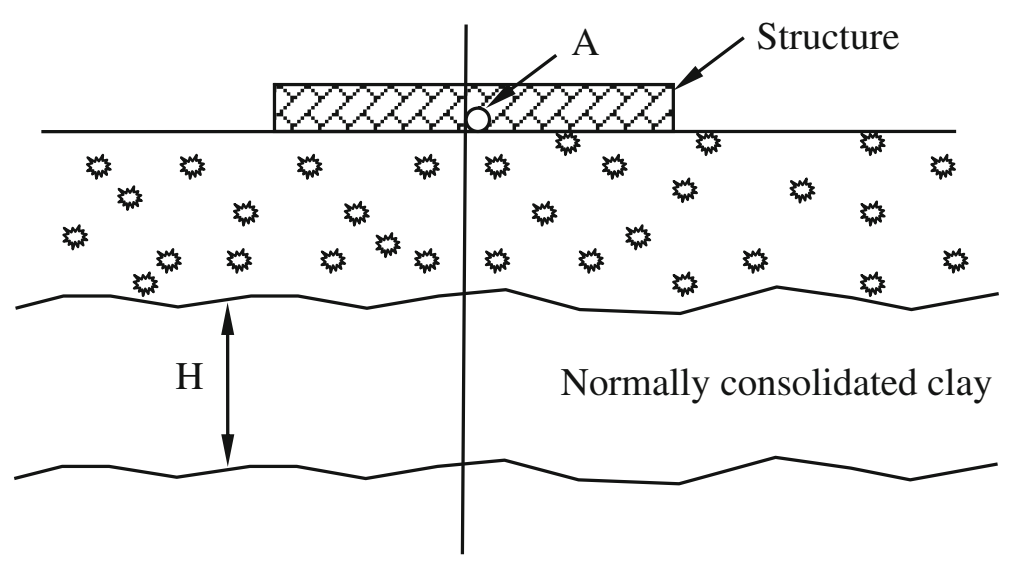

Figure 11. Soil profile (example 3).

increases. The fuzzy frequencies are skewed to the left as is the fuzzy number for the modulus of elasticity, since the frequency solution is directly related to the modulus of elasticity.

\subsection{Soil settlement}

The settlement of a point $A$ in figure 11 due to the construction of a structure is primarily caused by the consolidation of the clay layer. Suppose the contribution of settlement due to secondary consolidation is negligible. For normally loaded clay, the settlement $S$ is given by

$$
S=\frac{C_{c}}{1+e_{0}} H \log \frac{p_{0}+\Delta p}{p_{0}}
$$

where $C_{c}$ is the compression index of the clay; $e_{0}$ is the void ratio of the clay layer before loading; $H$ is the thickness of the clay layer; $p_{0}$ is the original effective pressure at point $B$

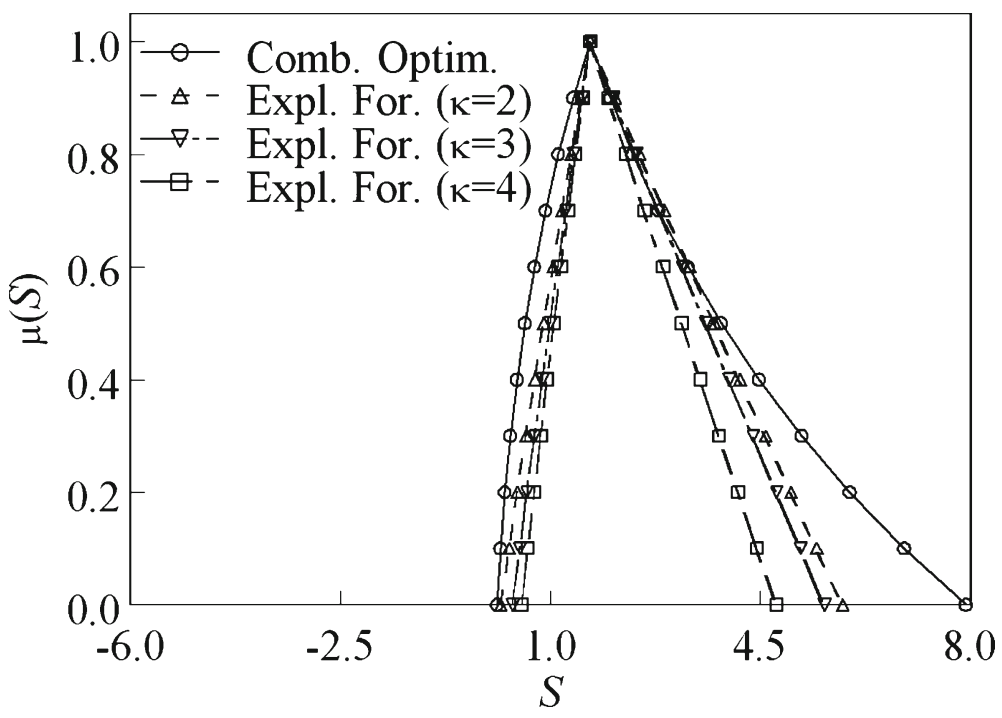

Figure 12. Possibility distributions of soil settlement (example 3). 

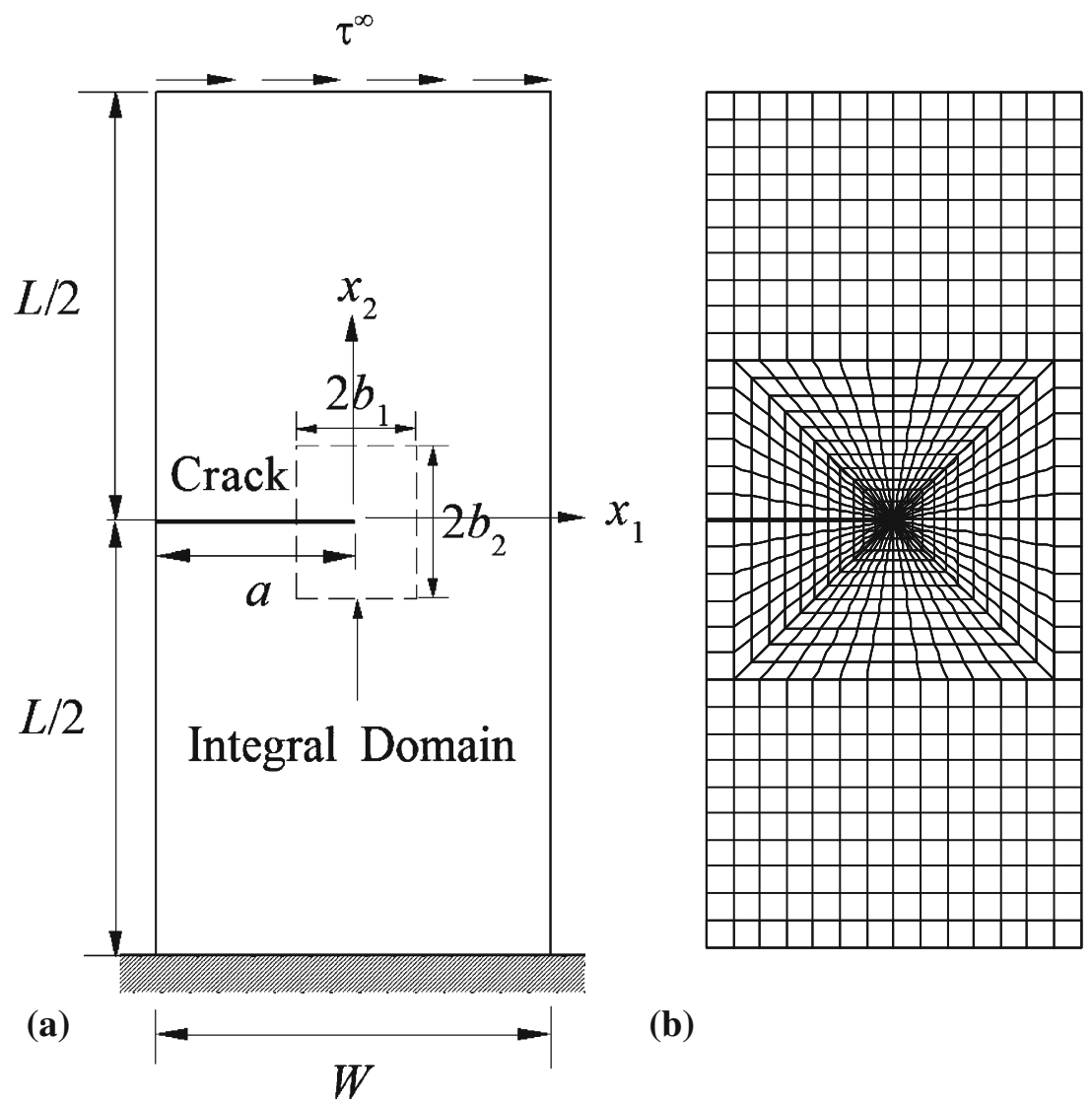

(b)

Figure 13. Edge-cracked plate (example 4); (a) Geometry, loads, and domain size; and (b) FEM discretization.

before loading; and $\Delta p$ is the increase in pressure at point $B$ caused by the construction of the structure; 'log' denotes logarithm to the base 10. Because of the non-uniform thickness and lack of homogeneity of the clay layer, the settlement predicted by the empirical formula could be subject to uncertainty in predicted settlement. This is a practical engineering example having the triangular fuzzy variables as follows. $C_{c}=[0.099,0.396,0.693], e_{0}=[0.6545,1.19,1.7255]$, $H=[142.8,168.0,193.2], p_{0}=[3.162,3.72,4.278]$, and $\Delta p=[0.2,0.5,0.8]$.

The possibility distributions of the fuzzy soil settlement obtained using the proposed firstorder HDMR based explicit formulation and the crude vertex method fuzzy analysis in conjunction with combinatorial optimization are presented in figure 12. Similar to the previous example, the effects of number of sample points and the order of approximation $\kappa$ are examined. Again the proposed first-order HDMR based explicit formulation estimates a narrow bound for the response and with increase in the order of approximation $\kappa$ the bounds for the response are observed to become still narrower.

\subsection{Edge cracked plate}

This example involves the edge-cracked plate shown in figure 13(a), which is fixed at the bottom and subjected to far-field shear stress of $\tau^{\infty}$ unit applied on top. Due to the far-field shear 
stress $\tau^{\infty}$, the plate is subjected to mixed-mode deformation involving fracture modes I and II (Anderson 1995). The mixed-mode stress-intensity factors $K_{I}(\boldsymbol{x})$ and $K_{I I}(\boldsymbol{x})$ are calculated using an interaction integral method (Yau et al 1980). The plate has length $L=16$ units, width $W=7$ units and crack length $a$. The elastic modulus is assumed to follow a hyperbolictangent gradation function which can be expressed with respect to the local $\left(x_{1}, x_{2}\right)$ Cartesian coordinates,

$$
E\left(x_{1}\right)=\frac{E_{1}+E_{2}}{2}+\frac{E_{1}-E_{2}}{2} \tan h\left(\eta x_{1}\right)
$$
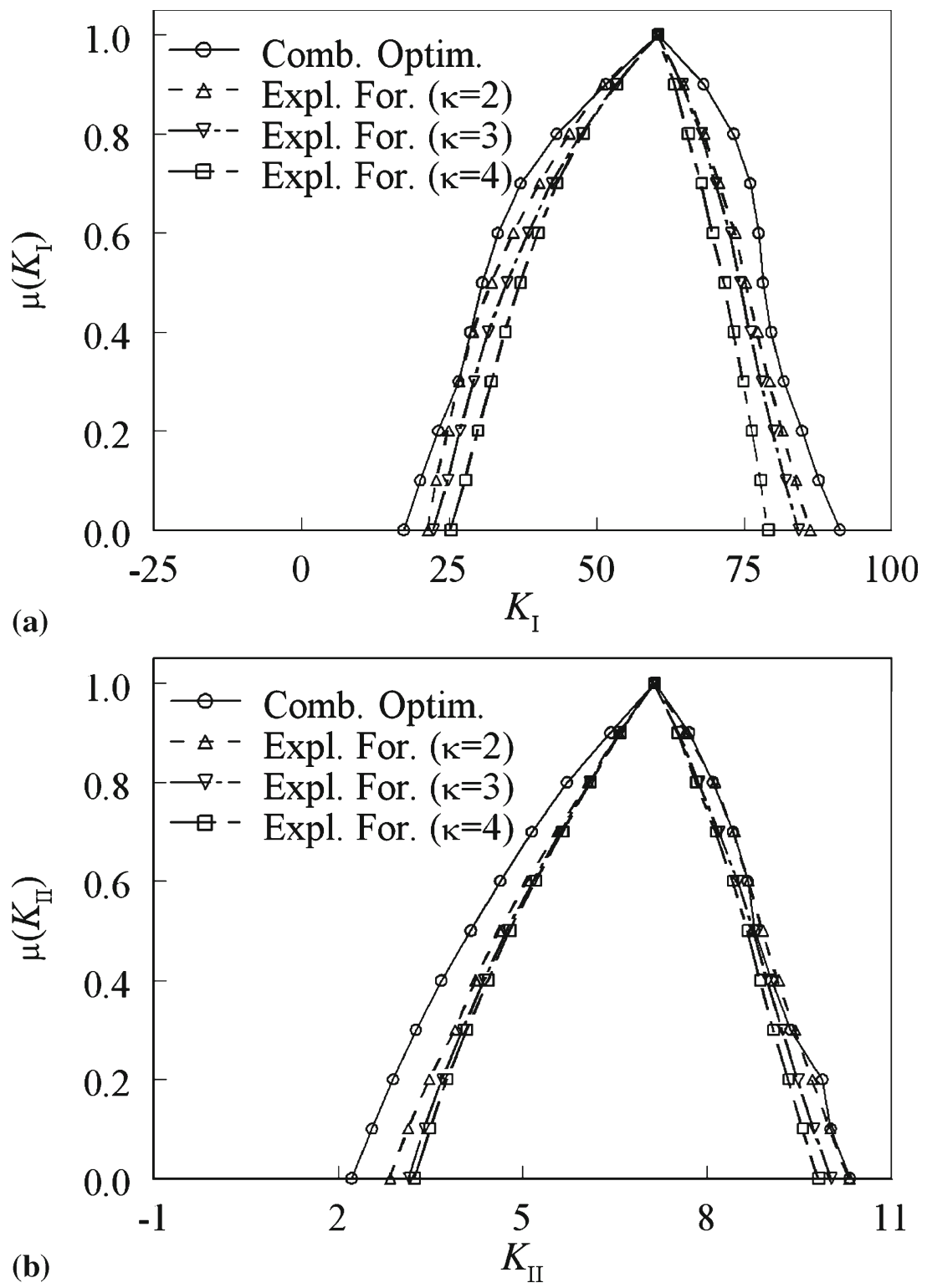

Figure 14. Possibility distributions (example 4); (a) $K_{I}$; and (b) $K_{I I}$. 
where $E_{1}=E\left(x_{1}=-0.5 \mathrm{~W}\right), E_{2}=E\left(x_{1}=0.5 \mathrm{~W}\right), \eta$ is gradation parameter. $E_{1}=[0.5,1.0$, 1.5], $E_{2}=[2.5,3.0,3.5], \eta=[5,7.5,10], \tau^{\infty}=[0.5,1.0,1.5]$ and $a=[3.0,3.5,4.0]$ are assumed to be fuzzy variables having triangular membership functions. A plane strain condition was assumed. A domain size $2 b \times 2 b$ ( $b=3.0$ units), as shown in figure 13(a) is used to calculate the mixed-mode SIFs. FEM discretization involved 2711 nodes, 832 8-noded quadrilateral elements, and 48 focused quarter-point 6-noded triangular elements, as shown in figure 13(b). The possibility distributions of the mixed-mode SIFs $K_{I}$ and $K_{I I}$ are shown in figures 14(a) and (b) respectively. Again the proposed first-order HDMR based explicit formulation estimates a narrow bound for the response and with increase in the order of approximation $\kappa$ the bounds for the response are observed to become still narrower.

\section{Conclusions}

In this paper a practical approach based on HDMR for analysing the response of structures with fuzzy parameters is presented. The proposed methodology involves integrated finite element modelling, HDMR based response surface generation, and explicit fuzzy analysis procedure. The uncertainties in the material, geometric, loading and structural parameters are represented using fuzzy sets. To facilitate efficient computation, a HDMR based response surface generation is employed for the approximation of the fuzzy finite element response quantity.

In the proposed method, using HDMR the response is expressed in a separable closed-form expression as a linear combination of the fuzzy variables through the definition of intervening variables, then using the transformation technique the bounds on the response at each $\alpha$-level are obtained. The advantage of this approach is that the bounds of the fuzzy response at an $\alpha$-level can be obtained by solving the resulting set of interval equations once. This approach is mathematically elegant and not computationally expensive. The use of transformations facilitates the determination of the minimum and maximum values of the fuzzy variables that correspond to the extreme values of the response at a particular $\alpha$-level without the use of optimization techniques. Moreover, this is semi-analytical procedure making use of deterministic finite element software and hence there is little room for errors. The proposed explicit formulation is free from the problem associated with dependency of the fuzzy variables, unlike most of the reported explicit formulations which are plagued with practical problems like the possibility of obtaining unbounded, unrealistic and non-unique solutions if the dependent relations between the fuzzy variables and the constrained fuzzy operation procedures are not considered in the formulation.

\section{Nomenclature}

$\begin{array}{ll}A & \text { Area } \\ a & \text { Crack width } \\ C_{c} & \text { Compression index of the clay } \\ \boldsymbol{c} & \text { Reference point } \\ E & \text { Elastic modulus } \\ e_{0} & \text { Void ratio of clay } \\ g(\boldsymbol{x}) & \text { Performance function } \\ \tilde{g}(\boldsymbol{x}) & \text { Approximated performance function } \\ g_{0}=g(c) & \text { Response at reference point } \\ g_{i}\left(x_{i}\right), g_{i_{1} i_{2}}\left(x_{i_{1}}, x_{i_{2}}\right) & \text { HDMR component functions }\end{array}$




\begin{tabular}{|c|c|}
\hline$K_{I}, K_{I I}$ & Stress intensity factors \\
\hline$L, W, H$ & Length, width, height \\
\hline$m$ & Number of $\alpha$-cuts \\
\hline$N$ & Number of variables \\
\hline$n$ & Number of sample points \\
\hline$P$ & Concentrated load \\
\hline$p_{0}$ & Original effective pressure \\
\hline$\Delta p$ & Increase in pressure \\
\hline$S$ & Soil settlement \\
\hline$s$ & Order of component function \\
\hline$U$ & Strain energy \\
\hline$u$ & Displacement \\
\hline$x$ & Uncertain variable vector \\
\hline$x_{i, \alpha}^{I}=\left[\underline{x}_{i}, \bar{x}_{i}\right]_{\alpha}$ & Interval variable \\
\hline$\left[x_{i L}, x_{i M}, x_{i U}\right]$ & Fuzzy variable \\
\hline$z_{i}$ & Intervening variable \\
\hline$\alpha$ & Confidence level \\
\hline$\beta_{i}$ & Coefficient \\
\hline$\gamma_{i}$ & Constant \\
\hline$\mu_{\tilde{x}}(x)$ & Membership function \\
\hline$\eta$ & Gradation parameter \\
\hline$\kappa$ & Order of approximation \\
\hline$\phi_{j}\left(x_{i}\right), \phi_{j_{1} j_{2}}\left(x_{i_{1}}, x_{i_{2}}\right)$ & Interpolation functions \\
\hline $\mathcal{R}_{2}, \mathcal{R}_{3}$ & Residual errors \\
\hline$\Re$ & Real coordinate space \\
\hline$\rho$ & Mass density \\
\hline$\tau_{\infty}$ & Far-field shear stress \\
\hline$\omega$ & Natural frequency \\
\hline$\tilde{x}$ & Fuzzy set \\
\hline
\end{tabular}

\section{References}

Adduri PR, Penmetsa RC 2008 'Confidence bounds on component reliability in the presence of mixed uncertain variables,' Int. J. Mech. Sci. 50(3): 481-489

Akpan UO, Koko TS, Orisamolu IR, Gallant BK 2001a 'Practical fuzzy finite element analysis of structures,' Finite Elem. Anal. Des. 38: 93-111

Akpan UO, Koko TS, Orisamolu IR, Gallant BK 2001b 'Fuzzy finite element analysis of smart structures,' Smart Mater. Struct. 10: 273-284

Alefeld G, Herzberger J 1983 Introduction to interval computations, (New York, USA: Academic Press)

Anderson TL 1995 Fracture mechanics: Fundamentals and applications, Boca Raton, Florida: CRC Press Inc.

Chen C 1996 Fuzzy logic and neural network handbook, Computer engineering series, New York: McGraw-Hill

Chen L, Rao SS 1997 'Fuzzy finite element approach for the vibration analysis of imprecisely defined systems,' Finite Elem. Anal. Des. 27: 69-83

Chowdhury R, Rao BN, Prasad AM 2008 'High dimensional model representation for piece wise continuous function approximation,' Commun. Numer. Methods Eng. 24(12): 1587-1609

Dessombz O, Thouverez F, Laîné JP, Jézéquel L 2001 'Analysis of mechanical systems using interval computations applied to finite element methods,' J. Sound Vib. 239(5): 949-968 
Dhingra AK, Rao SS, Kumar V 1992 'Nonlinear membership function in multi-objective fuzzy optimization of mechanical and structural systems,' AIAA J. 30: 251-260

Donders S, Vandepitte D, Van de Peer J, Desmet W 2005 'Assessment of uncertainty on structural dynamic responses with the short transformation method,' J. Sound Vib. 288(3): 523-549

Dong WM, Shah HC 1987 'Vertex method for computing functions of fuzzy variables,' Fuzzy Sets Syst. 24(1): 65-78

Dong WM, Wong FS 1987 'Fuzzy weighted averages and implementation of the extension principle,' Fuzzy Sets Syst. 21(2): 183-199

Drucker H, Burges CJC, Kaufman L, Smola A, Vapnik V 1997 'Support vector regression machines,' Adv. Neural Inf. Process. Syst. 9: 155

Elishakoff I 1995 'Essay on uncertainties in elastic and viscoelastic structures: from A.M. Freudenthal's criticism to modern convex modelling,' Comput. Struct. 56(2): 871-895

Farkas L, Moens D, Vandepitte D, Desmet W 2008 'Application of fuzzy numerical techniques for product performance analysis in the conceptual and preliminary design stage,' Comput. Struct. 86(10): 10611079

Gersem HD, Moens D, Desmet W, Vandepitte D 2007 'Interval and fuzzy dynamic analysis of finite element models with superelements,' Comput. Struct. 85(5-6): 304-319

Haldar A, Mahadevan S 2000 Reliability assessment using stochastic finite element analysis, 1st Edition, (New York: John Wiley \& Sons, Inc.)

Hansen E 1992 Global optimization using interval analysis, New York, USA: Marcel Dekker, Inc.

Hanss M 2002 'The transformation method for the simulation and analysis of systems with uncertain parameters,' Fuzzy Sets Syst. 130(3): 277-289

Harvey RL 1994 Neural network principles, NJ, USA: Prentice-Hall Inc.

Jones DR, Schonlau M, Welch WJ 1998 'Efficient global optimization of expensive black-box functions,' J. Glob. Optim. 13(4): 455-492

Lernout EC, Pellissetti M, Pradlwarter H, Schueller GI, Soize C 2006 'Data and model uncertainties in complex aerospace engineering systems,' J Sound Vib. 295(3-5): 923-938

Li G, Rosenthal C, Rabitz H 2001a 'High dimensional model representations,' J. Phys. Chem. A 105: 7765-7777

Li G, Wang SW, Rabitz H 2001b 'High dimensional model representations generated from low dimensional data samples-I. mp-Cut-HDMR,' J. Math. Chem. 30(1): 1-30

Moens D, Vandepitte D 2004 'Non-probabilistic approaches for non-deterministic dynamic FE analysis of imprecisely defined structures,' Proceedings of the International Conference on Noise and Vibration Engineering, ISMA, Leuven, 3095-3119

Möller B, Graf W, Beer M 2000 'Fuzzy structural analysis using $\alpha$-level optimization,' Comput. Mech. 26(60): 547-565

Moore RE 1979 Methods and applications of interval analysis, Philadelphia, USA: SAIM Publication

Muhanna RL, Mullen RL 2001 'Uncertainty in mechanics problems interval-based approach, J. Eng. Mech., ASCE, 127(6): 557-566

Munck MD, Moens D, Desmet W, Vandepitte D 2008 'A response surface based optimisation algorithm for the calculation of fuzzy envelope FRFs of models with uncertain properties,' Comput. Struct. 86(10): $1080-1092$

Pellissetti MF, Schueller GI 2007 'The effects of uncertainties in structural analysis,' J. Struct. Eng. Mech. 25(3): 311-330

Qiu Z, Elishakoff I 1998 'Antioptimization of structures with large uncertain-but-non-random parameters via interval analysis,' Comput. Methods Appl. Mech. Eng. 152(3-4): 361-372

Rao BN, Chowdhury R 2008 'Probabilistic analysis using high dimensional model representation and fast Fourier transform,' Int. J. Comput. Methods Eng. Sci. Mech. 9(6): 342-357

Rao SS and Berke L 1997 'Analysis of uncertain structural systems using interval analysis,' AIAA J. 34(4): $727-735$

Ross TJ 2004 Fuzzy Logic with Engineering Applications, Second Edition, Chichester: John Wiley \& Sons Ltd. 
Schueller GI, Pradlwarter HJ, Koutsourelakis P 2004 'A critical appraisal of reliability estimation procedures for high dimensions,' Probab. Eng. Mech. 19(4): 463-474

Sobol IM 1993 'Sensitivity estimates for nonlinear mathematical models,' Math. Model. Comput. Exper. 1: 407-414

Sobol IM 2003 'Theorems and examples on high dimensional model representations,' Chem. Reliab. Eng. Syst. Saf. 79(2): 187-193

Soize C 2009 'Nonparametric probabilistic approach of uncertainties for elliptic boundary value problem,' Int. J. Numer. Methods Eng. 80(6-7): 673-688

Valliappan S, Pham TD 1995 'Fuzzy logic applied to numerical modeling of engineering problems,' Comput. Mech. Adv. 2: 213-281

Yau JF, Wang SS, Corten HT 1980 'A mixed-mode crack analysis of isotropic solids using conservation laws of elasticity,' J. Appl. Mech. 47: 335-341

Zadeh L 1965 'Fuzzy sets,' Inf. Control 8(3): 338-353 